\title{
Research Paper: Revisiting the International Classification of Functioning, Disability and Health, a Comprehensive Model for Exploring Disablement in Low- and Middle- income Countries: A Narrative Overview
}

\author{
Surajo Kamilu Sulaiman $^{1 *}$ (D), Ashiru Hamza Mohammad² ${ }^{(D)}$, Aminu Alhassan Ibrahim ${ }^{3}$ (D), Sham'unu Isa Abdu ${ }^{4}$, Bashir Kaka ${ }^{1}$ (iD)
}

1. Department of Physiotherapy, Faculty of Allied Health Sciences, College of Health Sciences, Bayero University Kano, Nigeria.

2. Godiya Disability Inclusion and Development Initiative (GDID), Dutse, Jigawa State, Nigeria.

3. Department of Physiotherapy, Muhammad Abdullahi Wase Teaching Hospital, Kano State, Nigeria.

4. Department of Physiotherapy, General Hospital Kazaure, Nigeria.

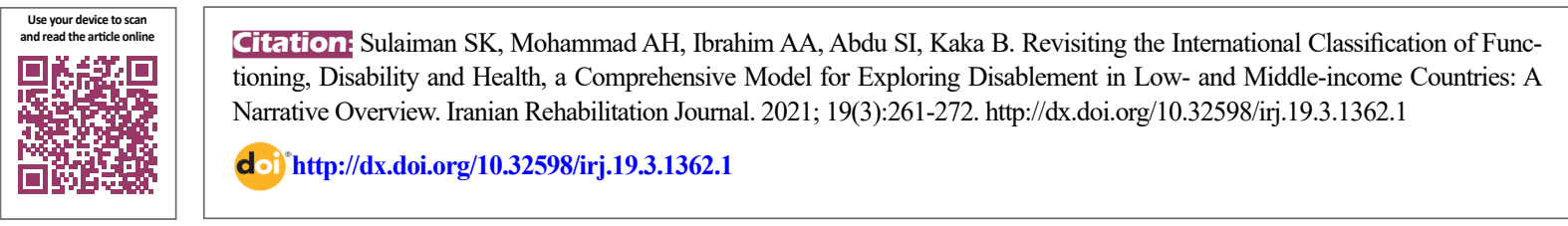

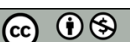

Article info:

Received: 05 Mar 2021

Accepted: 04 Aug 2021

Available Online: 01 Sep 2021

Keywords:

International Classification of Functioning, Disability, and Health (ICF), Models of disability, Disability evaluation, Conceptual framework, Lowand middle-income countries

\section{A B STRACT}

Objectives: To overcome the inherent limitations of the medical and social models of disability, the World Health Organization developed the first international conceptualization of disability: the International Classification of Functioning, Disability, and Health (ICF). Despite the ICF's robustness, it is still underutilized in research and practice in Low- and MiddleIncome Countries (LMICs). This article discusses the applications, strengths, limitations, and unique considerations when using the ICF to explore disabilities in LMICs like Nigeria.

Methods: A literature search was conducted in Medline, CINAHL, Web of Science, AMED, and Google Scholar. Articles were selected if they reported on any of the development, structure, applications, strengths, and limitations of the ICF. The review draws from the selected articles using a narrative discussion.

Results: The literature search yielded 22 articles that met the eligibility criteria. The ICF is a classification of components of health and functioning, which views disability as an outcome of a dynamic interaction between a person's health condition and contextual factors. The ICF was developed cross-culturally; thus, it is applicable in various socio-environmental contexts. However, despite its comprehensiveness, the ICF is criticized for lack of clear theoretical underpinnings, overlapping and redundant components, and absence of systematized personal factors.

Discussion: The ICF has brought a significant paradigm shift in the measurement of disability by explicitly recognizing the role of contextual factors in the incapacitation process and placing all health conditions on an equal footing. Hence, stakeholders in disability research and practice in LMICs need to prioritize the ICF over other disablement models.

\section{* Corresponding Author:}

Surajo Kamilu Sulaiman, PT., PhD.

Address: Department of Physiotherapy, Faculty of Allied Health Sciences, College of Health Sciences, Bavero University Kano, Nigeria. Tel: +23 (480) 35179594

E-mail:sksulaiman.pth@buk.edu.ng 


\section{Highlights}

- The medical and social models of disablement dominated the conceptualization of disability in the $20^{\text {th }}$ century. However, these models were criticized for narrowing disability into one of its aspects.

- To overcome the limitations of the medical and social models, the WHO developed the first international conceptualization of disability, the ICF.

- The ICF was develop cross-culturally to provide a classification system of components of health and functioning, and envisaged disability as an outcome of a dynamic interaction between person's health condition and contextual factors.

- Despite the robustness of the ICF, it is seldom employed compared to other more limited models of disablement in low-income setting like Nigeria. Hence researchers and other relevant stakeholders should prioritize the ICF when addressing disability issues.

\section{Plain Language Summary}

Disability is a complex phenomenon that is difficult to define and measure. Previously, the medical and social models of disability were commonly used to operationalize and assess disability. These models were criticized by scholars and other stakeholders for portraying disability as strictly a medical problem (medical model) or social problem (social model). To address the challenges of operationalizing disability, the World Health Organization developed the International Classification of Functioning Disability and Health (ICF). The ICF synthesizes what is true about disability in both medical and social models, to envisage disability as a product of dynamic interaction between a person's health condition and contextual factors. Hence the ICF is known as the biopsychosocial model of disability. Despite the robustness of the ICF, it is seldom employed to study or address disability issues in low-income settings like Nigeria. This review explores the strength and limitations of the ICF, to justify the need for stakeholders to prioritize the ICF over other more limited models of disability when addressing disability issues in low-income settings.

\section{Introduction}

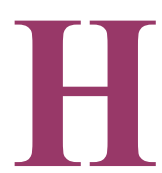

istorically, persons with disabilities have been overlooked in international development and global health circles because they were incorrectly seen as individuals whose lives are defined by their medical and rehabilitative needs [1]. This notion has its roots in the medical model of disability, which objectifies people based on their medical and pathological conditions. The medical model reiterates the provision of healthcare and rehabilitation services to individuals based on the degree and severity of their impairment. One of the significant drawbacks of the medical model is that it leads to 'paternalism, pathologization, and benevolence' [2]. Researchers criticized the medical model for viewing disabled individuals as pitiable and disempowered, thus casting a negative image of people with disabilities rather than seeing disability as a political, social, and environmental problem [3].
The social model of disability arises in response to the growing global disability rights movement, which has fostered a clearer understanding that the constraints faced by persons with disabilities reflect social, cultural, and economic barriers and are not inherently part of living with a disability. The social model has broadened its scope to include a human rights component, which comprises the right to health care, education, and social participation. Some of the identified weaknesses of the social model are neglecting impairment as an essential aspect of disabled people's life, absolute rejection of the medical approach that implies impairment as a problem, and the assumption that disabled people are oppressed [2]. Both the medical and social models of disability dominated the conceptualization of disablement in the 20th century; however, researchers criticized these models for narrowing disability to one of their aspects [3].

The World Health Organization (WHO) introduced the International Classification of Impairments, Disabilities and Handicaps (ICIDH) in the 1980s [4]. The ICIDH was meant for trial purposes, aimed at analyzing, de- 
scribing, and classifying the consequences of diseases. The ICIDH was the first internationally-shared conceptualization of disability and the first international system to classify the consequences of diseases [3]. Researchers criticized the ICIDH, especially for its biomedical orientation and disregard for the role of the physical, social, and attitudinal environments in the disablement process $[3,5-7]$ diseases and their consequences. These considerations have generated various conceptual models, some of which share the same focus and point of arrival, the so-called 'Disablement Process'.

Among the models that were developed, two stand out, which were drafted and disseminated under the aegis of the WHO, namely the International Classification of Impairments, Disabilities and Handicaps (ICIDH). Subsequent upgrades and modifications of the ICIDH throughout the 1990s resulted in the development and endorsement of the International Classification of Functioning, Disability, and Health (ICF) by the World Health Assembly in 2001 [8]. The ICF is based on the principles of universality, parity, neutrality, and consideration of context. The ICF comprises two parts. The first part deals with the components of functioning and disability, which are body structure, body function, activities, and participation. The second part covers the contextual factors, including personal and environmental factors [8, 9].

The ICF is based on a coherent biopsychosocial paradigm of disability, which integrates the biomedical and social models of disablement into a holistic spectrum [8]. This biopsychosocial perspective has made the ICF a holistic tool for exploring disability in low- and middleincome countries (LMICs), where disability is still primarily viewed via the medical prism [10]. Based on the ICF, functioning and disability are viewed as outcomes of a dynamic interaction between the person's health condition and contextual factors $[5,6,8]$. Thus, the ICF integrates the role of the context into the disablement process. This contextual orientation has made the ICF an instrument of choice for exploring disability, especially in LMICs, where social and environmental factors often contribute to the disablement process than the health condition alone. The ICF is a classificatory framework of health and the health-related state and a conceptual model for understanding the disablement process [11]. However, despite the remarkable qualities of the ICF, it is still rarely used as a conceptual framework or classification system to explore disablement in LMICs $[12,13]$ and for most countries, these questions are not based on either the ICIDH or the ICF.
Disability status is ascertained mainly through use of impairment screens that include a list of a few severe impairments. Some studies have shown the use of the ICIDH and also the ICF in the development of questions on disability $[12,13]$. This may be attributed mainly to guidelines in the United Nations census recommendations regarding use of the ICF framework and terminology to develop disability screening questions. This narrative overview aims to present the applications, strengths, weaknesses, and unique features of the ICF, making it the model of choice for disability research and practice in LMICs.

\section{Materials and Methods}

This article employs an unstructured narrative overview that draws from the extant literature based on critical reflection (Ferrari, 2015; Green, Johnson, \& Adams, 2006).

\section{Search strategy}

A literature search was conducted in five databases: MEDLINE, CINAHL, Web of Science, AMED, and Google Scholar, using the following search terms: international classification of functioning, disability and health, disability evaluation, theoretical model, biopsychosocial theory, conceptual framework, utility of the ICF, limitations of the ICF, strengths of the ICF, and structure of the ICF.

\section{Eligibility criteria}

Articles were included if they reported on the development, applications, uses, relevance, limitations, strengths, or theoretical underpinnings of the ICF. However, articles were excluded if they were not published in English, not available online, and reported as either experimental studies, observational studies, or qualitative studies.

\section{Study selection and data extraction}

Relevant information was extracted from the included articles based on the eligibility criteria, specifically, a report or discussion on the development, structure, applications, utility, relevance, limitations, strengths, or theoretical underpinnings of the ICF.

\section{Results}

We identified 48 articles from the database search (Figure 1). After the removal of 10 duplicates, 22 articles met our eligibility criteria. We synthesized the applications, strengths, limitations, and unique considerations when us- 
ing the ICF to explore disablement in the context of LMICs. These issues are discussed in the following section.

\section{Discussion}

This section discusses the applications, strengths, limitations, and unique features of the ICF, based on what was reported in the present literature, to justify the suitability and conformability of the ICF for low- and middle-income countries such as Nigeria.

\section{Applications of the ICF}

As a universal framework, the ICF has various applications across different settings, ranging from LMICs in Africa and Asia to High-Income Countries (HICs) in Europe and North America. The ICF provides users with a basis for understanding and describing disability experiences and a systematic classification that allows for the coding of all components of health and functioning [14]. Broadly speaking, the ICF is used in research, clinical practice, education of healthcare professionals, community support services, population-based studies, educational systems, policy, program development, advocacy, and empowerment $[4,8]$. The literature has reported various applications of the ICF across the domains mentioned above. For instance, Mbogoni (2003) reviewed the application of the ICIDH and the ICF in population surveys in LMICs between 1990 and 2000, using data available from the United Nations Disability Database [13].

The study reported a considerable variation between the methods of disability evaluation used in population surveys. Most evaluations were conducted using concepts drawn from the medical model. However, following the endorsement of the ICF, disability surveys and population censuses conducted around the year 2000 have tended to utilize some of the concepts described by the ICF [13]. This finding reveals the dominance of the medical model of disability and the over-medicalization of disability in LMICs, where little attention is paid to the role of the sociocultural and physical environmental contexts in the disablement process.

Medicalization of disability persists in LMICs like Nigeria. For example, in a national baseline survey of persons with disabilities conducted in Nigeria, neither the ICF nor the social model was utilized in the process, and the survey drew mainly from the medical and charity models of disability [15]. The recommendation of the ICF to member countries by WHO is yet to take effect fully. In another literature survey on the use of the
ICF, the article reported that a limited number of studies utilize the ICF in LMICs compared to high-income countries [12]. This underutilization of the ICF could be due to the limited emphasis that is given to disability and disability research in addition to the medicalization of disability in LMICs. Although the ICF impacts how data on disability are conceptualized, collected, and analyzed, especially in HICs, its use in LMICs is limited and needs to be actively encouraged. For example, in Nigeria, disability is commonly viewed in terms of charity and welfare, neglecting the contributions of the environmental context [16]. Thus, the ICF could be employed to make a phenomenal shift in disability research and practice, which could change the way disability and disabilityrelated issues are viewed and addressed in Nigeria.

In both HICs and LMICs, users have employed the ICF as a conceptual model for research, intervention, management planning, linking pre-existing outcome measurement instruments, core sets development, data gathering, and psychometric analysis [12]. The ICF has also been used at population, social, and healthcare levels [11]. At the population level, the ICF is employed in surveys of general and specific populations to collect health and disability information. It is also used in data compilation and analysis, development of disability survey modules and questions, as well as policy development and monitoring. At the social and healthcare levels, the ICF is used as a standard reference for health and disability-related legislation, evaluation of needs and services, and certification of disability [11].

The ICF represents one of the most significant paradigm shifts in rehabilitation science and practice [1719]. The ICF is a prominent model among rehabilitation specialists and has been employed in several ways such as a conceptual framework in research (to describe the relationship among different components of functioning, to develop and test hypotheses, to explicate research findings, etc.) and as a clinical tool for the assessment, planning, management, and evaluation of rehabilitation interventions and outcomes in clinical practice and governance [17, 18, 20-23]. The following section describes the strengths of the ICF, thereby distinguishing the ICF from other models of disability.

\section{Strengths of the ICF}

The ICF introduced significant innovations that sealed inherent loopholes within the dominant concepts of disablement: the medical and social models $[6,14]$. This outcome was possible because of the coherent theoretical and conceptual foundations upon which the ICF has 

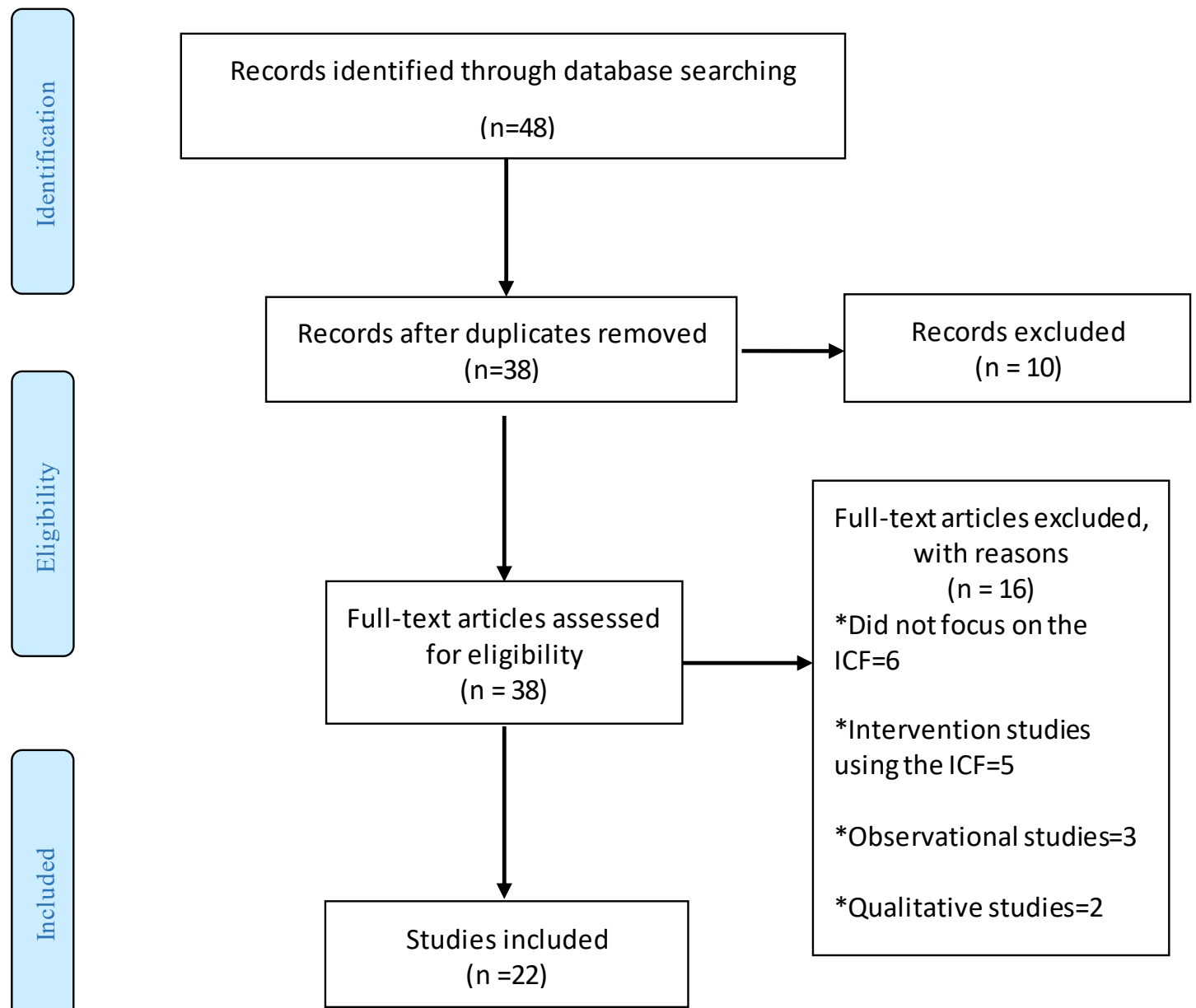

Full-text articles excluded, with reasons

$(n=16)$

*Did not focus on the ICF $=6$

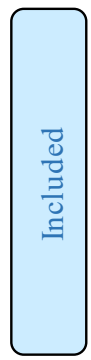

*Intervention studies using the $\mathrm{ICF}=5$

*Observational studies=3

*Qualitative studies $=2$

Figure 1. Flow Chart of included studies

Iranian Rehabilitation Journa

been developed. As a conceptual model, the ICF provides an extensive framework and a unified, standardized language for describing health and health-related states at both individual and societal levels [11]. Unlike the biomedical and social models of disablement, the ICF synthesizes what is true and valuable in both models. In the ICF, disability is viewed as a consequence of dynamic interaction between biological, personal, and social factors $[6,14]$. The ICF paved the way for users to view disability differently by taking personal and environmental features into account. In this regard, the ICF has a great potential to transform the conceptualization and measurement of disability in LMICs, which may lead to a better approach to describing disability-related interventions such as social and healthcare services.

The main feature of the ICF lies in its universality, parity, neutrality, and consideration for the context as an inseparable component of the disablement process. The ICF is universal as it is meant for all people, irrespective of health condition or functional status, and supports the develop- ment and implementation of contextualized interventions $[8,11]$. The interventions can be channeled to provide for the needs of all people irrespective of disability status, especially in LMICs where the distribution of basic social amenities may be lopsided. The parity denotes that the ICF does not discriminate health conditions based on etiology and provides a neutral ground for comparing various health conditions and health-related states $[8,11]$. This issue is essential, especially in the context of LMICs like Nigeria, where people with disabilities are often categorized and labeled based on their health problems - due to over-medicalization of disability_during routine evaluation.

The principle of neutrality refers to the neutral language used to describe components of functioning and disability within the ICF [8]. For example, in some LMICs like Nigeria, derogatory labels are sometimes used to label people with disabilities [24]. The ICF could be used to provide neutral language for proper characterization of disability. The inclusion of environmental and personal 
factors within the ICF makes it a holistic framework for describing disability experience [22].

Unlike previous models of disability, the ICF provides the opportunity to extensively explore the experience of disability by taking the unique features of the individual as well as the physical, social, and attitudinal environment into cognizance. This is paramount for explicating disablement, especially in the context of LMICs, where negative societal attitudes and institutional barriers are likely to restrict persons with disabilities from full participation in mainstream society [1]. In Nigeria, for example, some of the significant challenges persons with disabilities encounter include environmental barriers like inaccessible buildings and transport, institutional barriers such as the absence/failure to implement disability policies, and social barriers like negative attitudes and beliefs towards disability [16]. Here, the ICF can offer users a suitable framework for addressing disability and other related issues to make better the welfare of all individuals, including persons with disabilities.

The ICF is a biopsychosocial model of disability, which integrates components of health and functioning into a unified, coherent spectrum, mapping out the dynamic relationship between the various components of health and functioning [11]. Imms (2006) noted that one distinguishing feature of the ICF lies in its ability to provide a model, which articulates the complex and dynamic relationship between the multiple elements of an individual's life to determine health and health-related outcomes [25]. This issue is relevant, especially in the context of LMICs, where environmental factors are usually disregarded in the disablement process. The biopsychosocial perspectives expressed in the ICF recognize the interactive influence of physical, psychological, and social factors on health and the relevance of understanding the individual's viewpoint concerning their health and wellbeing [5, 6, 8]. Besides, Nordenfelt (2006) has described the notion of health expressed in the ICF as the most outstanding contribution to the healthcare system, marking a focal shift from disability to ability or capacity/capability [26]. The ICF promotes a solid scientific base for understanding functional states associated with various health conditions and provides a coding system for comparing data across various contexts [27].

As a classification system, Üstün et al. (2003) identified features that made the ICF an essential instrument for identifying and measuring the effectiveness of rehabilitation services in various contexts, including LMICs [14]. These features include detail, functional profiling, and intervention targeting [14]. The level of detail in the ICF refers to the hierarchical organization of the individ- ual components of health and functioning within the ICF. All items are arranged hierarchically, permitting users to pick an appropriate level of granularity that suits their purpose. Also, the items are conceptually defined with clear descriptions that are consistent and measurable where possible to permit real-life application. Besides, the inclusion and exclusion criteria are provided to allow users to differentiate between the various components of health. This clarity permits considerable flexibility and multiple applications in research and practice, especially in the context of LMICs, where several factors other than medical conditions are likely to influence an individual's functioning and health [14].

Functional profiling entails how the ICF classifies functioning and disability. Components of body structure, body function, activities, and participation are grouped in domains and subdomains. The body structure and functions are arranged according to body systems and anatomical body parts, while activities and participation are grouped. The activities and participation are distinguishable based on the application of qualifierscapacity and performance. The ICF allows the functional profiling of individuals, thus moving away from a disease-based classification system and reflecting the general principle of parity upon which it was developed [14]. Functional profiling using the ICF recognizes environmental and personal factors as independent components of the disablement process. This issue is paramount in the context of LMICs, where environmental and attitudinal influences probably restrict individuals from participation in life activities [16, 28].

Intervention targeting entails how users can identify a disabling factor and apply appropriate interventions. Preintervention evaluation using the ICF taxonomy allows for comparing outcomes and monitoring interventions directed at the individual or the environment [14]. The ICF supports assessment strategies closely related to selecting treatment goals, tracking clinical changes, exploring treatment outcomes, and measuring performance over time [27]. The ICF has been described as a living document that permits continuous upgrade of its contents based on the conceptual and philosophical assumptions it was developed [11]. This provides room for the modification of the ICF to specific contexts such as LMICs, where sociocultural influences may largely determine the construction of disability. Despite these distinguishing features, the ICF has limitations. The reported limitations of the ICF are described in the next section. 


\section{Limitations of the ICF}

Various limitations of the ICF were reported in the literature. These limitations include lack of a robust theoretical underpinning, clarification between components of activities and participation, systematization of personal factors, subjective dimensions such as wellbeing and quality of life, and temporal domain [22]. Imrie (2004)argued that theoretically, ICF does not clearly delineate the nature and contents of functioning and disability, which may limit its potential to inform users about the dynamic relational nature of functioning and disability [5]. Hence, researchers suggest that some aspects of ICF require further conceptual clarification and development. These aspects include the contents of the biopsychosocial theory, the meaning and implications of the ICF universal principle, and redefining the nature of impairment [18]. Moreover, despite the comprehensive nature of the ICF, there is a lack of clarity about its essential assumption — viewing disability as a dynamic process rather than a unique feature. This notion is more explicit in other disablement models such as Nagi's model and the Institute of Medicine model [3]. Although it is essential to acknowledge any shortcoming in the ICF, these particular limitations are unlikely to have any significant impact on the utility of the ICF in LMICs, where models with grave limitations, such as the medical model and the tragedy/charity model, are commonly employed [16].

Researchers contend that the ICF is comprehensive and cumbersome $[20,25]$. The time required to complete its checklist makes it impracticable in its current form, and therefore it requires further modification and adaptation $[20,25]$. This is especially true in the context of LMICs, where both infrastructural and manpower resources are likely to be inadequate, and the comprehensibility of the ICF could render it time-consuming for users. Although the ICF takes cognizance of personal and environmental factors, it needs to account for the interaction between personal and environmental factors too if it is to be applicable in research and practice. Moreover, it is essential to distinguish between modifiable and non-modifiable personal and environmental factors in the ICF. These factors are crucial to the rehabilitation process, especially where the physical and social environmental contexts are likely to be more disabling than the individual's health condition [20].

Researchers have identified several issues expressed by ICF users, such as missing or overlapping codes, inadequate granular codes, and lack of standardization on the use of qualifiers for activity and participation - users, generally apply the qualifiers inconsistently. This deficit may lead to inconsistency and difficulty comparing dis- ability information across various contexts $[12,29]$. The absence of systematized personal factors is one of the most major limitations of the ICF, especially in LMICssince the identification of personal factors is also critical to the disablement process - where attitudes and cultural beliefs largely influence an individual's personality. The ICF does not cover disease or diagnosis and lacks a positive terminology in relation to impairment and disease (Wade \& Halligan, 2003). Although this state may be viewed as a limitation, it must be recalled that ICF was not designed to classify disease or diagnosis, and it employs neutral concepts to describe functioning and health [11]. Thus, this particular shortcoming may not affect its utility in LMICs.

ICF does not account for temporal factors, for example, an individual's stage of life or illness. This may limit its applicability where time is required to monitor the level of functioning or severity of the disability. Moreover, the ICF appears to be externally driven and does not account for phenomena arising within the individual, for example, the individual's free will or volition. Besides, the ICF does not distinguish or acknowledge a possible difference between the individual's perspective and that of an external examiner [30]. These limitations are likely to undermine its utility in LMICs since individual perspectives largely contribute to the subjective experience of functioning and disability.

Nordenfelt (2006) opposes the ICF's emphasis on the actual performance of an action, which is entailed by a performance qualifier. Instead, he suggests using the opportunity and an opportunity qualifier since contextual factors are likely to enable or disable an individual and may significantly influence the disablement process [26]. This condition is true, especially in the context of LMICs. Besides, Nordenfelt's proposition would better reflect the notion of disability and functioning as an outcome of the interaction between health conditions and contextual factors. Besides, the ICF fails to account for the subjective dimension of functioning and disabilitysubjective wellbeing and quality of life-which could permit a thorough understanding of the individual's inner experience of disability [31]. However, the ICF was not designed for such a purpose, and hence this matter may not be an apparent limitation. The following section describes some unique features of the ICF, thus providing a clear rationale for its applicability in LMICs.

\section{Unique features of the ICF}

The ICF has many unique qualities, including being multinational, contextual, interactive, and comprehen- 
sive, which place it on top of other models for exploring disablement. Although some of these features have been discussed earlier in this paper, each is elaborated explicitly in the following subsections.

\section{Multinational}

The ICF was developed via international collaboration and validated in over 70 countries [11]. This feature makes it a unique framework with potential applicability in various contexts, including LMICs. Before introducing the ICF, disability evaluation was usually done using several heterogeneous concepts, which lacked clarity, consistency, and agreement, thus, making disability data comparison difficult across nations. Besides, disability policies were generally based on either the biomedical model or the social model [1]. The ICF brought a significant paradigm shift in the conceptualization and measurement of disability. To date, the ICF is the most widely accepted model of disability globally [11].

\section{Contextual}

As a biopsychosocial model, the inclusion of contextual factors (environmental and personal factors) as significant and independent contributors to the disablement process is one of the most distinguishing features of the ICF. The environmental factors constitute the physical, social and attitudinal factors in which people live and conduct their lives. The ICF provides an extensive framework for describing all possible aspects of the environment $[32,33]$. The personal factors denote the particular background of the individual's life and comprise features of the individual that are not part of the health condition. These factors include age, gender, race, lifestyle, coping, social background, level of education, profession, behavior, character, individual psychological assets, and other related characteristics. All or any of these factors could play a vital role in the disablement process [29].

\section{Interactive}

The ICF is a framework for describing the person-environment interaction and the outcomes of this interaction. Several elements are involved in this dynamic interaction that ought to be considered. These elements include the individual and their characteristics, the external environmental context, the individual's perception of their environment, and the process of interaction between these elements and the outcome of this interaction (i.e., functioning at the body level, individual level, and societal level) $[32,33]$. As reflected in the ICF, the personenvironment interaction is complex and dynamic. The environmental influence on functioning and disability is hierarchical, ranging from immediate or proximal to generic or distal. The immediate environment reflects factors that have a direct effect on an individual, for example, accessible public buildings and transport systems, while the generic environment reflects factors that have an indirect influence on the individual, for example, public policies and laws which govern the environmental context. Both proximal and distal environmental effects are crucial to the disablement process and were not mirrored in previous conceptualizations of disability $[32,33]$

One caveat is those umbrella concepts such as rural context, poverty, socioeconomic conditions, social capital, social support, and development that typically feature in the context of LMICs but did not appear as items in the ICF list of environmental factors. However, the level of detail and granular codes within the ICF environmental domain are likely to provide sufficient information to make up for the absence of these umbrella concepts [33].

The advent of the ICF has brought a significant change in disability management by expanding interventions to include the environment. Using the ICF, interventions can be directed at the individual or society to remedy either functional decrement or, most importantly, remove environmental barriers and enhance facilitators [32, 33]. It is essential, especially in the context of LMICs like Nigeria, where the medical and charity models of disability largely govern the management of disability [16]. The ICF can offer a befitting alternative to address disability issues by accounting for all the relevant domains in the disablement process.

\section{Comprehensive}

The ICF provides a comprehensive and standardized language for the description of functioning and disability across various contexts. The ICF conceptualizes human actions in terms of the intrinsic capacities of the individual to perform the actions (capacity) and the performance of the actions in the individual's actual environment (performance). Most aspects of human functioning are equally represented in the ICF. The domain of body structure and body function provides a detailed description of functioning at the level of the individual's body system. The domain of activities and participation provides an extensive description of functioning at the individual level and the individual's functioning within his environment. The environmental domain provides a detailed description of the features of the physical, social, and attitudinal environment $[8,9]$. 
The ICF is concerned with the objective description of functioning and disability. However, the ICF can be used for subjective assessment or evaluation of components of health and health-related states. Functioning and disability concepts within the ICF are arranged hierarchically in a systematic manner. The distinction between the perspectives of capacity and performance is an essential feature of the ICF, which reflects the role of the environment in the disablement process. The ICF can be incorporated into the ICD-10 for purposes such as national and international epidemiological surveys, health system evaluation, health and disability policy, empowerment, and advocacy [9, 11, 14, 34]. Hence, the ICF is a relevant tool, especially in the LMICs setting, where data comparison across contexts may be required to inform policy, research, and practice.

\section{Limitations of the review}

This paper is an unstructured narrative overview. Hence it carries all the limitations of a narrative overview $[35,36]$. For example, the information provided is purely narrative and based on the authors' reflections. However, this article contributes to the present literature by recasting the ICF in light of its utility in LMICs and recollecting the justification for prioritizing the ICF over more limited disablement models such as the medical and charity models.

\section{Conclusion}

The ICF is designed to provide a common approach for conceptualizing and measuring functioning and disability regardless of the setting. The ICF explicitly recognizes the roles of socio-environmental contexts in the disablement process and places all health conditions on similar footage within the framework. With the ICF, disability is not seen as a personal feature but a product of a dynamic interaction between health conditions and contextual factors. Thus, the ICF is the most widely accepted conceptualization of disability, which provides a standardized framework and language of communication to professionals and other users. The unique features of the ICF make it an invaluable tool for understanding human experience in various contexts. Despite the compelling advantages of the ICF, some limitations are still discernable within its theoretical underpinnings and structural constructs. However, the ICF surpasses both the medical and social models of disability by synthesizing only what is true in the two models to portray the disablement process on a biopsychosocial spectrum. Hence, this narrative overview recommends applying the ICF in LMICs like Nigeria, where less robust disablement models such as the charity model are still being used to explore disablement.

\section{Ethical Considerations}

Compliance with ethical guidelines

There were no ethical considerations to be considered in this research.

\section{Funding}

This research did not receive any grant from funding agencies in the public, commercial, or non-profit sectors.

\section{Authors' contributions}

Conceptualization: Surajo Kamilu Sulaiman; Methodology: Surajo Kamilu Sulaiman and Ashiru Hamza Mohammad; Literature search and information extraction: Surajo Kamilu Sulaiman and Ashiru Hamza Mohammad; Writing original draft: Surajo Kamilu Sulaiman; Review and editing: All authors.

\section{Conflict of interest}

The authors declared no conflict of interest.

\section{Acknowledgments}

We appreciate the feedback provided by Dr. Vincent G. DePaul, School of Rehabilitation Therapy, Queen's University, Canada.

\section{References}

[1] World Health Organization (WHO). World report on disability [Internet]. 2011. Available from: https://www.who. int/disabilities/world_report/2011/report.pdf

[2] Pfeiffer D. The conceptualization of disability. In: Barnartt SN, Altman BM, editors. Exploring Theories and Expanding Methodologies: Where We Are and Where We Need to Go (Research in Social Science and Disability). Bingley: Emerald Group Publishing Limited; 2001. [DOI:10.1016/S14793547(01)80019-1]

[3] Masala C, Petretto DR. From disablement to enablement: Conceptual models of disability in the $20^{\text {th }}$ century. Disability and Rehabilitation. 2008; 30(17):1233-44. [DOI:10.1080/09638280701602418] [PMID]

[4] World Health Organization (WHO). International classification of impairments, disabilities, and handicaps [Internet] 1980. Available from: https://apps.who.int/iris/bitstream/ handle/10665/41003/9241541261_eng.pdf;jsessionid 
[5] Imrie R. Demystifying disability: A review of the international classification of functioning, disability, and health. Sociology of Health \& Illness. 2004; 26(3)287-305. [DOI:10.1111/ j.1467-9566.2004.00391.x] [PMID]

[6] Jette A. Toward a common language for function, disability, and health. Physical Therapy. 2006; 86(5):726-34 [DOI:10.1093/ptj/86.5.726] [PMID]

[7] Kearney PM, Pryor J. The International Classification of Functioning, Disability and Health (ICF) and nursing. Journal of Advanced Nursing. 2004; 46(2):162-70. [DOI:10.1111 /j.1365-2648.2003.02976.xrJAN2976 [pii]] [PMID]

[8] World Health Organisation (WHO). Towards a common language for functioning, disability and health ICF [Internet] 2002. Available from: https://www.who.int/classifications/ icf/icfbeginnersguide.pdf

[9] World Health Organization (WHO). How to use the ICF: A practical manual for using the International Classification of Functioning, Disability and Health (ICF) [Internet]. 2013. Available from: https://www.who.int/classifications/drafticfpracticalmanual.pdf

[10] Me A, Mbogoni M. Review of practices in less developed countries on the collection of disability data. In: Altman $\mathrm{BM}$, Barnartt SN, editors. International Views on Disability Measures: Moving Toward Comparative Measurement. Amesterdam: Emerald Group Publishing; 2006. p. 63-87. [DOI:10.1016/S1479-3547(05)04006-6]

[11] Kostanjsek N. Use of The International Classification of Functioning, Disability and Health (ICF) as a conceptual framework and common language for disability statistics and health information systems. BMC Public Health. 2011 11 Suppl 4 (Suppl 4):S3. [DOI:10.1186/1471-2458-11-S4-S3] [PMID] [PMCID]

[12] Jelsma J. Use of the international classification of functioning, disability and health: A literature survey. Journal of Rehabilitation Medicine. 2009; 41(1):1-12. [DOI:10.2340/165019770300] [PMID]

[13] Mbogoni M. On the application of the ICIDH and ICF in developing countries: Evidence from the United Nations Disability Statistics Database (DISTAT). Disability and Rehabilitation. 2003; 25(11-12):644-58. [DOI:10.1080/096382803100013 7144] [PMID]

[14] Üstün TB, Chatterji S, Bickenbach J, Kostanjsek N, Schneider M. The international classification of functioning, disability and health: A new tool for understanding disability and health. Disability and Rehabilitation. 2003; 25(11-12):565-71. [DOI:10.1080/0963828031000137063] [PMID]

[15] Federal Ministry of Women Affairs \& Social Development. Report of the national baseline survey on Persons with Disabilities (PWDs) in Nigeria [Internet]. 2013 [Updated 2016 September 9]. Available from: www.womenaffairs.gov.ng

[16] Lang R, Upah L. Scoping study: Disability issues in Nigeria [Internet]. 2008 [Updated 2008 April]. Available from: https://studylib.net/doc/13390397/scoping-study--disability-issues-in-nigeria-final-report

[17] Rauch A, Cieza A, Stucki G. How to apply the International Classification Of Functioning, disability and health (ICF) for rehabilitation management in clinical practice. European Journal of Physical and Rehabilitation Medicine. 2008; 44(3):329-42. [DOI:10.1002/14651858.CD005954.pub2.Fundings.]
[18] Stucki G, Ewert T, Cieza A. Value and application of the ICF in rehabilitation medicine. Disability and Rehabilitation. 2002; 24(17):932-8. [DOI:10.1080/09638280210148594] [PMID]

[19] Wade D, Halligan P. New wine in old bottles: The WHOICF as an explanatory model of human behaviour. Clinical Rehabilitation. 2003; 17(4):349-54. [DOI:10.1191/0269215503cr619ed] [PMID]

[20] Bartlett DJ, Macnab J, Macarthur C, Mandich A, MagillEvans J, Young $\mathrm{NL}$, et al. Advancing rehabilitation research: An interactionist perspective to guide question and design. Disability and Rehabilitation. 2006; 28(19):1169-76. [DOI:10.1080/09638280600551567] [PMID]

[21] McDougall J, Horgan K, Baldwin P, Tucker MA, Frid P. Employing the International Classification of Functioning, Disability and Health to enhance services for children and youth with chronic physical health conditions and disabilities. Paediatrics \& Child Health. 2008; 13(3):173-8. [DOI:10.1093/ pch/13.3.173] [PMID] [PMCID]

[22] McDougall J, Wright V, Rosenbaum P. The ICF model of functioning and disability: Incorporating quality of life and human development. Developmental Neurorehabilitation. 2010; 13(3):204-11. [DOI:10.3109/17518421003620525] [PMID]

[23] McDougall J, Wright V, Schmidt J, Miller L, Lowry K. Applying the ICF framework to study changes in quality-of-life for youth with chronic conditions. Developmental Neurorehabilitation. 2011; 14(1):41-53. [DOI:10.3109/17518423.2010.52 1795] [PMID] [PMCID]

[24] Ogundola OJ. Framing disability: A content analysis of newspapers in Nigeria [MA. thesis]. New York: Syracuse University; 2013._https://surface.syr.edu/ms_thesis/15

[25] Imms C. The international classification of functioning, disability and health: They're talking our language. Australian Occupational Therapy Journal. 2006; 53(2):65-6. [DOI:10.1111/j.1440-1630.2006.00575.x]

[26] Nordenfelt L. On health, ability and activity: Comments on some basic notions in the ICF commentary. Disability and Rehabilitation. 2006; 28(23):1461-5. [DOI:10.1080/09638280600925886] [PMID]

[27] Reed GM, Lux JB, Bufka LF, Trask C, Peterson DB, Stark $S$, et al. Operationalizing the international classification of functioning, disability and health in clinical settings. Rehabilitation Psychology. 2005; 50(2):122-31. [DOI:10.1037/00905550.50.2.122]

[28] Sango PN. Visible but invisible people living with disabilities in Nigeria [Internet]. 2013 [Updated 2013 November 13] Available from: https://books2africa.org/visible-but-invisible-people-living-with-disability-in-nigeria/

[29] Geyh S, Peter C, Müller R, Bickenbach JE, Kostanjsek N, Ústün BT, et al. The personal factors of the international classification of functioning, disability and health in the literature - a systematic review and content analysis. Disability and Rehabilitation. 2011; 33(13-14):1089-102. [DOI:10.3109/09638288 .2010.523104] [PMID]

[30] Wade D. Rehabilitation: A new approach. Part two: The underlying theories. Clinical Rehabilitation. 2015; 29(12):1145-54. [DOI:10.1177/0269215515601175] [PMID]

[31] Ueda S, Okawa Y. The subjective dimension of functioning and disability: What is it and what is it for? Disability and Rehabilitation. 2003; 25(11-12):596-601. [DOI:10.1080/0963828 031000137108] [PMID] 
[32] Chapireau F. The environment in the international classification of functioning, disability and health. Journal of Applied Research in Intellectual Disabilities. 2005; 18(4):305-11. [DOI:10.1111/j.1468-3148.2005.00269.x]

[33] Schneidert M, Hurst R, Miller J, Üstün B. The role of Environment in the International Classification of Functioning, Disability and Health (ICF). Disability and Rehabilitation. 2003; 25(11-12):588-95. [DOI:10.1080/0963828031000137090] [PMID]

[34] Bickenbach J, Posarac A, Cieza A, Kostanjsek N. Assessing disability in working age population a paradigm shift : from impairment and functional limitation to the disability approach [Internet]. 2015 [Updated 2015 June 18]. Available from: http://hdl.handle.net/10986/22353

[35] Ferrari R. Writing narrative style literature reviews. Medical Writing. 2015; 24(4):230-5. [DOI:10.1179/204748061 5Z.000000000329]

[36] Green BN, Johnson CD, Adams A. Writing narrative reviews for peer reviewed journals: Secrets of trade. Journal of Chiropodist Medicine. 2006; 5(30):101-17. [DOI:10.1016/ S0899-3467(07)60142-6] 
This Page Intentionally Left Blank 